\title{
Diagnostic applications of ultrasmall superparamagnetic particles of iron oxide for imaging myocardial and vascular inflammation
}

Short Title: Diagnostic applications of ultrasmall superparamagnetic particles of iron oxide

Ioannis Merinopoulos, MD, MSc ${ }^{\mathrm{a}, \mathrm{b}}$, Tharusha Gunawardena, $\mathrm{MD}, \mathrm{BSc}^{\mathrm{a}, \mathrm{b}}$, Colin Stirrat, $\mathrm{MD}$, $\mathrm{PhD}^{\mathrm{c}}$, Donnie Cameron, $\mathrm{PhD}^{\mathrm{a}, \mathrm{d}}$, Simon C Eccleshall, $\mathrm{MD}^{\mathrm{b}}$, Marc R Dweck, MD, $\mathrm{PhD}^{\mathrm{c}}$, David E Newby, MD, $\mathrm{PhD}^{\mathrm{c}}$, Vassilios S Vassiliou, $\mathrm{MD}, \mathrm{PhD}^{\mathrm{a}, \mathrm{b}}$

${ }^{a}$ Norwich Medical School, University of East Anglia, Norfolk and Norwich University Hospital, UK; ${ }^{b}$ Department of Cardiology, Norfolk and Norwich University Hospital, UK;

${ }^{c}$ BHF Centre for Cardiovascular Science, University of Edinburgh, 49 Little France

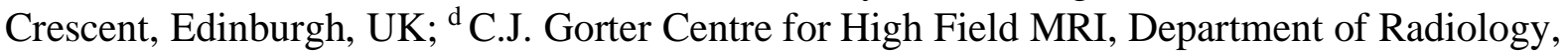
Leiden University Medical Centre, Leiden, The Netherlands

The authors have no competing interests.

\section{Address for correspondence}

Dr Vassilios S Vassiliou

2.06 Bob Champion Research \& Education Building

Norwich Medical School, University of East Anglia

Norwich, NR4 7TJ

Email: v.vassiliou@uea.ac.uk

Telephone: +44 (0)1603 592534

@vass_vassiliou 


\begin{abstract}
Cardiovascular magnetic resonance (CMR) is at the forefront of non-invasive methods for the assessment of myocardial anatomy, function and most importantly tissue characterization. The role of CMR is becoming even more significant with an increasing recognition that inflammation plays a major role for various myocardial diseases such as myocardial infarction, myocarditis and takotsubo cardiomyopathy. Ultrasmall superparamagnetic particles of iron oxide (USPIO) are nanoparticles that are taken up by monocytes and macrophages accumulating at sites of inflammation. In this context, USPIO-enhanced CMR can provide valuable additional information regarding the cellular inflammatory component of myocardial and vascular diseases. Here, we will review the recent diagnostic applications of USPIO in terms of imaging myocardial and vascular inflammation, and highlight some of their future potential.
\end{abstract}

Keywords: Cardiovascular magnetic resonance, ultrasmall superparamagnetic particles of iron oxide, inflammation, macrophage

\author{
Abbreviations: \\ AE: Adverse Event \\ CMR: Cardiovascular Magnetic Resonance \\ FDG: Flurodeoxyglucose \\ hs-cTnI: high sensitivity cardiac Troponin I \\ ION: Iron Oxide Nanoparticles \\ LGE: Late Gadolinium Enhancement \\ MI: Myocardial Infarction \\ MRI: Magnetic Resonance Imaging \\ NSTEMI: Non ST Elevation Myocardial Infarction \\ PET: Positron Emission Tomography \\ STEMI: ST Elevation Myocardial Infarction \\ USPIO: Ultrasmall Superparamagnetic Particles of Iron Oxide
}

\title{
Highlights:
}

- Ultrasmall superparamagnetic particles of iron oxide (USPIO) have enabled in vivo visualization of cellular inflammation, whose central role in the pathophysiology of multiple cardiovascular processes is increasingly recognized.

- USPIO-enhanced magnetic resonance imaging (MRI) has recently substantially improved our knowledge and understanding of several cardiovascular diseases.

- USPIO-enhanced MRI is a promising method with the potential to provide valuable diagnostic and prognostic information for cardiovascular diseases. 


\section{Introduction}

Inflammation is the main defense mechanism against infection or tissue injury, and is one of the body's response to extreme deviations from homeostasis induced by various stressful stimuli (1). Macrophages play a central role in these processes by detecting various types of stressors and responding accordingly with signals to orchestrate the inflammatory response (1). Ultrasmall superparamagnetic particles of iron oxide (USPIO) have been used successfully to assess cellular inflammation. These iron-oxide contrast agents are engulfed by tissue-resident phagocytic cells or infiltrating monocytes/macrophages and they generate signal inhomogeneities which can be detected by magnetic resonance imaging (MRI). Indeed, macrophages have an established role in all stages of atherogenesis as they underlie the development of coronary plaques, their progression to vulnerable plaques and eventual disruption (2). It was most recently demonstrated that inhibition of macrophage signaling and function reduced atherosclerosis in a mouse model, opening novel means for treating atherosclerosis (3). In addition, macrophages have divergent functions and also aid the healing process after myocardial infarction (2). Distinct macrophage subtypes with different polarization status are responsible for their diverse properties. Following a myocardial infarction, early pro-inflammatory macrophages (type 1) become polarized toward an antiinflammatory phenotype (type 2) later on. The balance between these macrophage subtypes plays a crucial role in myocardial repair and function following acute myocardial infarction (4). Therefore, determining the magnitude and nature of cellular inflammation is of paramount importance in guiding accurate diagnosis, monitoring disease progression, assessing therapeutic efficacy and determining risk stratification of cardiovascular disease. One potential non-invasive method for imaging and assessing cellular inflammation includes the use of USPIO-enhanced MRI. In this article, we will review its most recent diagnostic applications for imaging myocardial and vascular inflammation. 


\section{Iron oxide nanoparticles}

\section{Composition}

Iron oxide nanoparticles (ION) are formed by small particles of iron oxide with a coating derived from organic compounds. The core iron oxide consists of magnetite $\left(\mathrm{Fe}_{3} \mathrm{O}_{4}\right)($ most commonly) or maghemite $\left(\gamma-\mathrm{Fe}_{2} \mathrm{O}_{3}\right.$ or $\left.\alpha-\mathrm{Fe}_{2} \mathrm{O}_{3}\right)$, with a core diameter between 4-10 $\mathrm{nm}$ (5). The physiological properties of bare iron oxide particles are altered by the organic compound coating, which is often larger than the core iron oxide itself. Bare iron oxide is hydrophobic causing it to aggregate and undergo opsonisation (i.e. binding with plasma proteins) following injection into the bloodstream (6). Coating with organic compounds is therefore essential in order to increase their hydrophilicity and decrease opsonisation and the tendency of the particles to aggregate. These are important factors that determine the manner in which ION interact within the host body. In addition, the coating decreases their toxicity as it prevents the release of iron ions (6). Various compounds have been used for coating but polysaccharide dextran is most commonly used amongst FDA-approved ION-based contrast agents (7).

\section{$\underline{\text { Physiological properties }}$}

The size and coating of ION are two of the most important determinants of their properties (8). According to their size, ION can be classified into:

1) Very small superparamagnetic particles iron oxide with diameter $<20 \mathrm{~nm}$,

2) Ultrasmall superparamagnetic particles of iron oxide (USPIO) with diameter 20-50 nm

3) Small superparamagnetic particles of iron oxide with diameter $50-250 \mathrm{~nm}$

4) Micro-sized particles of iron oxide with diameter 1-8 $\mu \mathrm{m}(7)(9)$. 
Superparamagnetism is a property of USPIO resulting from their small size and crystalline nature. In the absence of an externally applied magnetic field, the net magnetization is zero as the magnetic orientations of single magnetic domains rotate free from thermal motion and cancel each other out. Application of an external magnetic field reorients the magnetic domains and results in a magnetic moment much greater than that of a paramagnetic substance. Termination of the external magnetic field, however, leads to immediate termination of their magnetic moment unlike larger ferromagnetic substances which retain their magnetic properties in the absence of a continuously applied external magnetic field (5) (10) (11).

Following intravenous administration, ION contrast agents remain in the intravascular space and ordinarily do not leak into the interstitium, provided that the endothelium is healthy and not affected by a pathological process (5). Resident macrophages of the reticuloendothelial system readily uptake ION from the blood circulation. Large ION ( $>1 \mu \mathrm{m}$ in diameter) tend to accumulate in the liver and lungs while USPIO are typically eliminated from blood via uptake by the liver, spleen and bone marrow (12). The half-life of each ION depends on its exact size and the chemical properties of the coating (13). In general, ION of a smaller size with a hydrophilic coating and neutral surface charge can escape immediate recognition from cells of the reticuloendothelial system and have a longer half-life compared to larger ION (7). USPIO for example may have a half-life up to 36 hours compared with a half-life of 2 hours for larger ION (9). The small size and long half-life of USPIO enable them to cross the capillary wall especially at sites where there is loss of endothelial integrity and increased capillary permeability (9) (14). Once accumulated in a tissue, ION cause local magnetic field inhomogeneities that shorten the $\mathrm{T} 2 / \mathrm{T} 2 *$ relaxation processes resulting in signal void (hypointense regions) on T2 and T2* weighted images (10). The effect of ION-based 
contrasts can be quantified by measuring the decrease in $\mathrm{T} 2 *$ value or an increase in $\mathrm{R} 2 *$ value $(\mathrm{R} 2 *=1 / \mathrm{T} 2 *)$ which are both frequently reported in the literature (15) (16). However, it is necessary to have paired MRI scans (pre- and post- ION-based contrast administration) to be able to measure accurately the change in $\mathrm{R} 2 *$ value from baseline. Given that IONbased contrast results in signal void (hypointense regions), the baseline scan increases the accuracy of image interpretation and artefact elimination. A delay between IONadministration and MRI is also necessary in order to allow enough time for the ION to circulate in the blood and concentrate at sites of interest. According to standard protocols, the second MRI takes place 24 hours after ION-based contrast administration (17). Careful consideration of the physiological properties of each individual ION is essential in order to understand the information that these nanoparticles are providing and to maximize their potential as biomarkers of cellular inflammation (18).

There are two proposed mechanisms to explain ION localization to sites of inflammation. According to the first, ION passively migrate across the endothelium at sites of increased permeability or loss of endothelial integrity, such as sites of inflammation (19). ION with smaller size have longer half-time, circulate longer in the blood circulation and therefore have more time to come in contact and potentially migrate across the endothelium. Following migration into the interstitium, ION are engulfed by tissue-resident macrophages via pinocytosis and thus become concentrated at sites of inflammation (9) (20). Alternatively, there are data to support a second mechanism, whereby ION are taken up by blood monocytes, stored within the reticuloendothelial system and subsequently transported to areas of inflammation. Montet-Abou et al. were the first to demonstrate in a mouse model that monocytes and macrophages labelled in vivo prior to myocardial ischemia-reperfusion injury can be tracked to the infarct area (21). Yang et al. similarly used a mouse model of 
myocardial infarction induced by left anterior descending artery ligation seven days after injection of microsized particles of iron oxide to demonstrate that the pre-labelled inflammatory cells mobilized to, and then infiltrated, the MI site (22); however these findings have not been replicated by others recently (23). Finally, human studies of patients with stroke undergoing USPIO-enhanced MRI have suggested that USPIOs are taken up solely by infiltrating macrophages rather than tissue resident macrophages (microglia cells) (24)(25). However, the lack of tissue biopsy in this study precludes definitive conclusions to be drawn.

It has recently been demonstrated that the scavenger receptor type A I/II (SR-AI/II) provides the predominant route of ferumoxytol uptake by mouse peritoneal macrophages in vitro and in vivo (26). The same study also demonstrated that SR-AI/II mediates uptake of ferumoxytol in vitro by $\mathrm{M} 1$ and $\mathrm{M} 2$ bone marrow derived macrophages (mouse) that express similar levels of SR-AI/II receptors (26), though these results have not been verified by all studies. We speculate that different cell surface receptors expressed by different macrophage subgroups affect the uptake or elimination of USPIO; subsequently leading to different concentration of USPIO within different macrophage subgroups.

\section{$\underline{\text { Magnetic resonance imaging of ION-based contrast agents }}$}

As stated previously, ION cause shortening of T2 and T2*, lending strong applicability to $\mathrm{T} 2 *$ imaging methods. The T2 shortening of USPIO is best visualized using $\mathrm{T} 2 *$-weighted gradient recalled echo pulse sequences, which are inherently sensitive to field inhomogeneities. While earlier ION studies used qualitative T2*-weighted imaging, recent studies have favored the quantitative $\mathrm{T} 2 *$ mapping approach recommended in the Society for Cardiovascular Magnetic Resonance consensus statement on parametric mapping (27): a multi gradient recalled echo sequence with eight echo times, ranging from 2 to $18 \mathrm{~ms}$. This 
sequence should preferably be applied with a black-blood preparation to reduce measurement bias and interobserver variability (28). Further, it is worth noting that the consensus suggests that $\mathrm{T} 2 *$ mapping be performed at $1.5 \mathrm{~T}$ in order to avoid the increased magnetic susceptibility artifacts at higher field strengths (27). Finally, the optimal range of echo times is not only determined by the USPIO agent but also by the uptake of USPIO in the tissue of interest. Tissue-specific echo times selected based on the expected $\mathrm{T} 2 *$ value will increase the accuracy of the decay curves (29).

It is important to consider that, as well as shortening T2 and T2*, ION also cause T1 shortening. This opens up applications for ION as contrast agents in magnetic resonance angiography (30), but it also has consequences in terms of $\mathrm{T} 2 *$ imaging pulse sequence parameters. The T1 shortening effect of ION is minimal when the nanoparticles are clustered together, as in this case there is a sharp increase in $\mathrm{r} 2$ relaxivity, leading to hypointensity on T2*-weighted images; however, in regions where the USPIO are relatively diffuse the T1shortening effect dominates, diminishing signal attenuation caused by $\mathrm{T} 2$ and $\mathrm{T} 2 *$ effects (31). This can lead to USPIO uptake being obscured when the Cardiovascular Magnetic Resonance (CMR) pulse sequence is highly T1-weighted, particularly for agents with lower r2-r1 ratios_-see Table 1 (32). Fortunately, this effect can be mitigated through use of lower flip angle radiofrequency pulses and longer echo and repetition times. The majority of CMR studies of ION to date have used Ferumoxytol; however, the use of other agents has little effect on the CMR pulse sequence design, excluding minor adjustments to the flip angle, echo time, repetition time, and range of echo times, based on the different relaxivities of the agent. On the other hand, different nanoparticle blood half-lives may permit further optimization of the post-administration CMR delay; however, this has not been studied in detail, and most studies to date have used a delay of 24 hours. 


\section{Diagnostic applications of iron oxide nanoparticles for myocardial imaging}

\section{Myocardial infarction}

Alam et al. were the first to publish an open-label pilot proof-of-concept study in 16 patients with STEMI treated with primary percutaneous coronary intervention (PCI) and stent implantation (33). Ten patients had three sequential cardiovascular magnetic resonance (CMR) scans at 3T within five days of admission at baseline, 24 and 48 hours following intravenous ferumoxytol (4 mg/kg; Feraheme, AMAG Pharmaceuticals) administration while six control patients had the same number of CMR scans but no ferumoxytol infusion. They demonstrated a significant increase in $\mathrm{R} 2 *$ in the infarct and peri-infarct area with an interesting but more modest increase in the remote 'healthy' myocardium, while there was no difference in the skeletal muscle, which acted as the control. Figure 1 shows an example of a patient one week post anterior myocardial infarction with significant USPIO uptake anteriorly and anteroapically.

The NIMINI-1 (Non-invasive myocardial inflammation imaging based on new molecular magnetic resonance imaging) study also investigated the possibility of visualizing the infarct and peri-infarct areas following acute coronary syndrome using a different USPIO (ferucarbotran, Resovist) with CMR (18). It included twenty patients with acute ST-elevation (STEMI) or non-ST-elevation myocardial infarction (NSTEMI) who had undergone prompt and successful PCI. The patients underwent a baseline pre-USPIO CMR within seven days of presentation and a second post-USPIO CMR 10 minutes $(n=2), 4$ hours $(n=2), 24$ hours $(n=10)$ or 48 hours $(n=6)$ after ferucarbotran administration. The results of that study were less helpful than anticipated as $\mathrm{T} 2 *$ sequences (pre- and post-ferucarbotran administration) did not offer any additional information when compared with standard late gadolinium 
enhancement (LGE). The absence of an effect was attributed to the low CMR field strength (1.5 T), the very small dose of ferucarbotran used (only $0.65 \mathrm{mg} \mathrm{Fe} / \mathrm{kg}$ ) and its short half-life ( $<15$ minutes) (18). The NIMINI-2 study had the same aim as NIMINI-1 trial; however, ferumoxytol was used instead of ferucarbotran (19). It included 14 patients with first STEMI who had undergone successful PCI within 24 hours. The patients had a baseline CMR at 1.5 $\mathrm{T}$ (median day 3 post-MI) and a second CMR 48 hours post-ferumoxytol administration. The authors detected a substantial drop in absolute $\mathrm{T} 2 *$ values not only in the infarct and periinfarct areas but also in the remote 'healthy' myocardium with minimal change observed in the skeletal muscle. Ex vivo analyses of cultured macrophages and peripheral blood mononuclear cells suggested that ferumoxytol was absorbed by infiltrating myocardial macrophages rather than by peripheral mononuclear cells that later translocated to the heart. However, there might have also been an additional direct ferumoxytol effect, due to USPIO partitioning into areas of myocardium with impaired capillary integrity (19).

Following these promising initial results, Stirrat et al. investigated the temporal changes in cellular inflammation and tissue edema post MI (16). They recruited 31 patients with STEMI or NSTEMI and performed repeat T2 mapping and repeat (ferumoxytol) USPIO-enhanced T2* CMR scans. They demonstrated that the infarct area had increased R2* compared with the remote myocardium until two weeks post MI. At the same time, native T2 values were higher in the infarct area compared to remote myocardium until three months after MI (Figure 2). Biopsies from three patients who subsequently underwent coronary artery bypass graft $(\mathrm{CABG})$ showed co-localization of iron staining with infiltrating macrophages in infarcted but not in non-infarcted areas. These data suggest that the most prominent contributor to USPIO enhancement into tissues is cellular inflammation in the myocardium rather than partitioning of USPIO into the tissues. However, an earlier scan a few hours after 
the infarction might have helped consolidate this as it is known that ferumoxytol effects are detectable as early as 6 hours after ferumoxytol administration, while differentiated human macrophages do not demonstrate ferumoxytol uptake before 24 hours of incubation. The data also suggest that day 2-3 post MI is the optimum time to image cellular inflammation, as the USPIO uptake in the infarct zone is at its peak. (18). The other important result of this study was that there was no time course variation in USPIO uptake in the peri-infarct and remote myocardium and that the amplitude of $\mathrm{R} 2 *$ change in the remote myocardium was less than that of blood pool. The peri-infarct area appeared to demonstrate increased USPIO uptake compared to remote myocardium early post MI although this observed trend did not reach statistical significance (16). Therefore, despite the intriguing results of the two aforementioned trials (19) (33), it appears likely that the increased signal in the remote myocardium is due to a diluted effect of blood-pool USPIO rather than macrophage uptake of USPIO within these tissues (16).

In further study, Lagan et al. investigated five patients with ischemic cardiomyopathy at mean of eight years after MI and four healthy volunteers who underwent CMR (1.5T) at baseline, two days and three days post-ferumoxytol infusion (34). They demonstrated that post-USPIO T2* values were lower in the infarcted myocardium compared to remote myocardium in patients with ischemic cardiomyopathy. This was an interesting result given that the aforementioned trial (16) had not shown a significant difference in the infarcted area when compared to the remote myocardium beyond two weeks post MI. The reason for the disparity between these two studies remains unclear but merits further clarification and research. It is possible that a difference in the degree of left ventricular systolic impairment of patient groups between these studies provides the explanation. However, we should emphasize that analysis of USPIO-enhanced CMR in patients with ischemic cardiomyopathy 
can be especially challenging due to increased artifacts in the interface between the blood pool and thin myocardium.

Finally, USPIO-enhanced CMR has also been used for the assessment of myocardial injury and inflammation related to coronary artery bypass graft (CABG) (15). The investigators assessed 87 patients undergoing CABG for stable coronary artery disease, with blood biomarkers of inflammation and myocardial injury, a baseline CMR (pre-CABG) and USPIO (ferumoxytol)-enhanced CMR at $3 \mathrm{~T}$ within 14 days of surgery. LGE was associated with the delayed 24-hour peak in plasma cardiac troponin I, but not systemic inflammation, myocardial inflammation or bypass time. The pan-myocardial R2* value was increased compared to healthy volunteers while the average $\mathrm{R} 2 *$ value for the three segments with the highest values from the 17-segment model was also increased compared to the panmyocardial R2* value. However, there was no correlation between USPIO uptake and plasma cardiac troponin I or cardiopulmonary bypass time, indicating that in the complex postCABG scenario, myocardial injury is not mediated solely by inflammation. Nevertheless, this was the first attempt to assess the elicited myocardial inflammation after CABG. Further studies are warranted to elucidate the determinants and long-term effects of increased cellular inflammation following CABG.

\section{Myocarditis}

CMR has a central role in diagnosing and monitoring myocarditis. The recently updated Lake Louise criteria demonstrate the additional benefit of the latest CMR sequences, including parametric mapping, and techniques in improving diagnostic accuracy for myocarditis (35). However, the updated CMR-based criteria aim to detect indirect indicators of myocarditis, such as myocardial edema and non-ischemic myocardial injury, rather than directly imaging 
cellular inflammation. Initial animal data in a rat model of experimental autoimmune myocarditis were very promising. Moon et al. were able to demonstrate in this preclinical model that magneto-fluorescent nanoparticle CMR effectively visualized myocardial inflammatory cellular infiltrates and provided more distinct images of inflammation compared to conventional CMR. The authors demonstrated that magneto-fluorescent CMR detected scattered, small and less severe foci of inflammation more accurately compared to conventional CMR and validated their findings with histological data (36).

To date, there is only one clinical study investigating the role of USPIO CMR in patients with acute myocarditis. Stirrat et al recruited 14 patients with suspected acute myocarditis and 10 volunteers who underwent T2, T2* mapping and LGE 3 T CMR with further T2* mapping CMR 24 hours post-ferumoxytol infusion, at baseline and three months later (37). Of the 14 recruited patients, 9 had confirmed acute myocarditis and were included in the study; two patients were excluded due to takotsubo cardiomyopathy and one for each of polymyositis, lung cancer and incompatible metallic implant. These 9 patients had typical CMR features of myocarditis in terms of LGE distribution and intensely high T2 values in regions of LGE. Despite the aforementioned promising preclinical work, there was no significant difference in USPIO uptake between patients and volunteers even within areas of LGE. The authors concluded that infiltrating macrophages do not contribute significantly to myocardial inflammation in myocarditis (Figures 3, 4). Even though, a previous study in patients with viral myocarditis and CMR guided biopsy, showed that LGE correlated well with a predominantly macrophage-rich inflammation (38), it has long been recognized that consensus on histological diagnosis is difficult even among experts (39). Stirrat et al. discussed various other possible explanations for these negative results, notably that 1) macrophages are not the predominant cell type in that patient group, and 2) there are inherent 
difficulties with image interpretation due to blooming artefacts being more dominant in the inferolateral walls, which is the usual site of myocarditis. Irrespective of the exact reason, based on the Stirrat et al study, there is no evidence to support clinical use of USPIO CMR in patients with myocarditis.

\section{Takotsubo cardiomyopathy}

There is currently one published clinical trial investigating the role of USPIO-enhanced CMR in patients with takotsubo cardiomyopathy. Scally et al. recruited 55 patients with takotsubo cardiomyopathy and 51 control subjects (40). The patients underwent $3 \mathrm{~T}^{31} \mathrm{P} C \mathrm{CMR}$ spectroscopy, T1 mapping, LGE and T2* mapping with repeat T2* mapping 24 hours after ferumoxytol infusion. The patients were assessed at baseline and at 5-month follow up. Compared to control subjects, patients with takotsubo cardiomyopathy had differences in the change of $\mathrm{T} 2 *$ (pre and post-ferumoxytol infusion) and native $\mathrm{T} 1$ values during the acute event in both ballooning and non-ballooning segments (Figure 5). That difference was no longer significant at 5 months. However, myocardial energetics, assessed by ${ }^{31} \mathrm{P}$ CMR spectroscopy, demonstrated that resting cardiac energetic status was markedly reduced acutely and that there was a continuing trend at 5 months. Previous studies had suggested a possible contributory role of edema and inflammation, as assessed by CMR, to the pathophysiology of takotsubo cardiomyopathy (41) (42). However, this study demonstrated for the first time a mechanistic pathway of macrophage-mediated myocardial cellular inflammatory response superimposed on myocardial edema. (40). Data on the optimum time for USPIO-enhanced CMR in patients with takotsubo is limited but given that all patients studied to date were assessed within 14 days of presentation, imaging within the first 2 weeks is suggested. 


\section{$\underline{\text { Vascular inflammation }}$}

It has become apparent over the last couple of decades that luminal stenosis is not the only factor predicting clinical vascular events. The development of novel imaging methods focusing on arterial wall inflammation offers a more elaborate assessment of atherosclerotic disease activity, aiming to predict cardiovascular events (43). USPIO-enhanced MRI of atherosclerosis was first attempted in the thoracic aorta of hyperlidemic rabbits about 20 years ago (44)(45). Since then, USPIO-enhanced MRI has been used successfully in clinical studies to image abdominal aortic aneurysms, carotid atherosclerosis and also monitor the effect of high-dose statin (46) (47) (48).

Tang et al. demonstrated in 2008 that there is no relationship between inflammation as assessed by USPIO-enhanced MRI and luminal stenosis in asymptomatic patients with carotid atherosclerosis, concluding that inflammation and stenosis are likely to be independent risk factors (49). A year later, the ATHEROMA trial demonstrated that highdose statin therapy over only three months reduced the USPIO-defined inflammation in patients with carotid atherosclerosis (46). Thus, USPIO-enhanced MRI can be utilized as an imaging biomarker to assess the inflammatory response of therapeutic interventions in patients with carotid atherosclerosis. Since then, inflammation of carotid atherosclerotic plaques has been evaluated by both USPIO-enhanced MRI and ${ }^{18}$ F-FDG PET/CT (Fluorodeoxyglucose positron emission tomography / computed tomography). Initial case reports showed that there was agreement between the two modalities in terms of the identified inflammatory activity (50). A more recent trial, however, which included only nine patients though, did not find a correlation between USPIO uptake and ${ }^{18}$ F-FDG uptake in carotid atherosclerotic plaques (51). This question will hopefully be clarified by a trial that is undergoing currently (NCT01674257) (52). This trial aims to assess carotid atherosclerosis 
using both USPIO-enhanced MRI and PET/CT (18F-FDG and 18F-NaF to image

inflammation and microcalcification) in patients who have had a stroke and are undergoing carotid endarterectomy. This is an intriguing and interesting trial as it will provide histological data and elucidate further the role of macrophages and micro-calcification in atherosclerosis (NCT01674257).

The first case report of USPIO uptake in abdominal aortic aneurysm (AAA) in humans was published in 2007 (53). Howard et al. described simultaneous USPIO uptake in the internal carotid artery and AAA. Since then, USPIO-enhanced MRI has been evaluated regarding its usefulness in predicting AAA expansion and repair. In a pilot study, Richards et al first demonstrated that USPIO-enhanced MRI identified cellular inflammation and suggested that it could be used as a tool to predict AAA expansion (54). The findings of this pilot study were further explored by the prospective multicenter open-label MA ${ }^{3}$ RS (MRI Using Ultrasmall Superparamagnetic Particles of Iron Oxide to Predict Clinical Outcome in Patients Under Surveillance for Abdominal Aortic Aneurysms) trial (55). The MA ${ }^{3}$ RS Investigators studied 342 patients with AAA using USPIO-enhanced MRI. USPIO enhancement was associated with increased rates of aneurysm expansion, rupture or repair, but it was not independent of known predictors of outcome such as baseline AAA diameter and smoking. Therefore, incorporating USPIO-enhanced MRI in a model did not improve outcome prediction beyond known risk factors. Nevertheless, it demonstrated for the first time that imaging cellular inflammation in patients with AAA can predict clinical events. Post hoc analyses did suggest that USPIO enhanced MRI could predict overall mortality in patients with larger aneurysms. The study also provided evidence that macrophage-mediated inflammation might be the pathophysiological mechanism linking smoking with disease progression in patients with AAA (55). Interestingly a recent study compared USPIO- 
enhanced MRI and ${ }^{18}$ F-FDG PET/CT for the detection of inflammation in AAA (56). It showed that there was only modest correlation between the two techniques, which identified different patterns and distribution of inflammation (56). This was attributed to the fact that USPIO-enhanced MRI and ${ }^{18} \mathrm{~F}$-FDG PET/CT detect different aspects of inflammation, macrophage phagocytic and glycolytic activity respectively, but the lack of histologic correlation prohibits definitive conclusions to be drawn (56). It is also possible that ${ }^{18} \mathrm{~F}$-FDG and USPIO target different macrophage subgroups, as has been suggested by in vitro data (57).

Similarly, encouraging results have been reported in the field of cerebral artery aneurysms. An initial pilot study suggested that early uptake of ferumoxytol (within 24 hours of infusion) predicted unstable cerebral aneurysm and demonstrated that inflammation was causally linked to aneurysm rupture (58). A small preliminary study from the same group, provided histologic and radiologic evidence that aspirin can attenuate the inflammatory response in the wall of human cerebral artery aneurysms. Following 3 months of treatment with aspirin, there was a reduction in ferumoxytol uptake compared to baseline, while histologic analysis showed COX-2, mPGES-1 and macrophages were reduced in the aspirin group compared with the control group (59) (60).

\section{Future directions}

\section{Macrophage subgroups}

Over the last few years, our understanding of macrophages, their various subtypes and their roles in steady-state and myocardial disease has advanced significantly. Various physiological stressors can produce different immune responses. It has been described for over a decade now that myocardial infarction elicits a biphasic inflammatory response (the 
first phase is characterized by pro-inflammatory monocytes and is followed by a second phase characterized by reparative monocytes), while a recent study suggested that takotsubo cardiomyopathy elicits a monophasic (CD16) monocyte response (61) (43). Recent data have also suggested that myocarditis is dominated predominantly by lymphocytes or neutrophils with macrophages playing a small part (37). Pro-inflammatory monocytes dominate during phase one (days one to five) after MI, promoting digestion of infarcted tissue and removal of necrotic debris; followed by reparative monocytes that dominate during phase two, promoting resolution of inflammation and myocardial repair (Figure 6) (61). The relative balance of these two phases is related to myocardial repair and function after MI (4). This has clinical implications, as it has already been demonstrated that the peak levels of pro-inflammatory CD16- monocytes were negatively correlated with the extent of myocardial salvage and recovery of left-ventricular function following MI (62). More recently, the combination of cell tracking and single cell RNA-sequencing has allowed characterization of many more transcriptionally distinct subgroups of macrophages in steady-state and post-MI models, adding a higher degree of complexity in categorization of macrophages beyond cell-surface markers (63).

In vivo imaging and distinction of the various macrophage subgroups is challenging, albeit with many potential clinical applications. So far, only in vitro studies have shown that it is possible to differentiate between pro-inflammatory M1 and anti-inflammatory M2 macrophages using USPIO-enhanced CMR. Laghi's group showed in vitro that it was possible to distinguish anti-inflammatory M2-polarised macrophages incubated with USPIO at $3 \mathrm{~T}$ based on their $\mathrm{T} 1$ and $\mathrm{T} 2 *$ signal intensities (64). Consistent with these results, Beer's group showed in vitro that it is possible to distinguish between pro-inflammatory M1 and anti-inflammatory M2 macrophages incubated with USPIO or gadolinium liposomes, based on their relaxation rates or susceptibility measurements (65). M1 macrophages had higher 
transverse relaxation rates and susceptibility compared with M2 and non-polarized M0 macrophages while M2 macrophages had significantly different susceptibility compared with M0 macrophages (65). These results are exciting and their translation to in vivo models is keenly awaited.

\section{Cardiomyocyte apoptosis}

Apoptosis is a form of programmed cell death, where cell death occurs as the end result of a specific sequence of steps, mediated by the intrinsic or extrinsic pathway. In contrast to necrosis, apoptosis represents a potentially reversible state for the cell. Therefore, accurate and timely imaging of cardiomyocyte apoptosis can be very valuable with clear therapeutic implications (66). CMR is considered to be the reference standard non-invasive method for tissue characterization post MI. LGE accurately demonstrates the necrotic infarcted myocardium while the area at risk is estimated via the more recently developed T1 and T2 mapping techniques (67). However, these techniques image indirect indicators of area at risk, such as edema, rather than cardiomyocyte apoptosis specifically. Phosphatidylserine (PS) and phosphatidylethanolamine (PE) usually reside on the interior layer of sarcolemma but are exteriorized and displayed on the cell surface during apoptosis, becoming attractive markers for imaging. Annexin-V is an endogenous protein that binds with high affinity to phosphatidylserine (68). AnxCLIO-Cy5.5 is a magneto-optical form of Annexin-V that retains its ability to bind apoptotic cells while being detectable by both optical fluorescence imaging and MRI (69). Sosnovik et al., in an elegant preclinical experiment using annexinbased nanoparticle (AnxCLIO-Cy5.5) to detect apoptosis and simultaneous delayedenhancement imaging to detect necrosis, demonstrated in vivo that the majority $(>70 \%)$ of the injured myocardium (within 4-6 hours of ischemia) was characterized only by the accumulation of AnxCLIO-Cy5.5 accumulation (most in mid-myocardium) without delayed 
enhancement (70). However, the fact that nanoparticles can also be taken up by macrophages might limit their suitability for purely detecting apoptosis in cardiac diseases where macrophages play a significant role. The use of radiolabeled molecules, such as ${ }^{99 m}$ Technetium-labelled Annexin V, to image apoptosis surpasses this problem but creates uncertainty regarding the functional consequences of anatomically imaged apoptosis (66). While waiting for clinical studies to define their role, there has been slow clinical translation of these apoptosis-imaging techniques into human studies.

\section{Clinical Practice}

Ferumoxytol (Rienso) was available at a competitive price for the treatment of iron deficiency anemia in Europe until it was withdrawn from the market in 2015 for commercial reasons and some uncertainty about possible hypersensitivity reactions (71) (72). The U.S. Food and Drug Administration issued a boxed warning about possible acute hypersensitivity reactions following 79 reported cases of anaphylaxis, including 18 fatalities during the therapeutic use of ferumoxytol (out of an estimated 1.2 million injections) (73) (74). Since then, its safety has been further supported. A recent, multicenter, double-blind randomized clinical trial comparing the safety of ferumoxytol versus ferric carboxymaltose for the treatment of iron deficiency anemia, specifically focused on hypersensitivity reactions including anaphylaxis (75). This large study of 2,000 patients did not raise any new safety signals of ferumoxytol compared to ferric carboxymaltose for moderate/severe hypersensitivity reactions including anaphylaxis and moderate/severe hypotension (75). The clinical safety of ferumoxytol for diagnostic purposes has also very recently been supported by registry data (74). In this multicenter MRI registry, more than 3,000 patients received an excess of 4,000 ferumoxytol injections between them, with only $1.8 \%$ mild adverse events (AE), $0.2 \%$ moderate $\mathrm{AE}$ and no severe or fatal AE (74). In addition, ferumoxytol's safety 
profile in patients with chronic kidney disease once again suggested that it can be a safe alternative to gadolinium-based contrast agents for the investigation of this group of patients (74). A number of recent studies have demonstrated that ferumoxytol can be used for vascular imaging in patients with chronic kidney disease without the safety concerns of gadolinium (76) (77) (78) (79).

Over the last few years, USPIO-enhanced CMR has been used successfully as a research tool for the non-invasive imaging of myocardial and vascular inflammation. Promising recent results may lead to USPIO entering clinical practice in the near future. That will be determined by its ability to provide accurate and cost-effective diagnostic and prognostic information over and above other non-invasive methods used currently. With that in mind, the results of the recent $\mathrm{MA}^{3} \mathrm{RS}$ trial are encouraging (55). It has opened the way for USPIOenhanced CMR to be tested in other inflammatory conditions with macrophage involvement such as vasculitis. Clearly non-invasive, radiation-free methods such as USPIO-enhanced CMR are favorable in clinical practice for both diagnosis and monitoring of disease processes. Therefore, the increasing amount of data supporting ferumoxytol's safety profile will help further expand its MRI applications, bringing it into the competitive arena of noninvasive imaging in clinical practice at a competitive price.

\section{Conclusion}

With an increasing recognition that inflammation underlies an expanding number of cardiovascular processes and diseases, the need to image it in a sensitive way becomes more important and clinically relevant. Over the last few years, USPIO-enhanced MRI has been used successfully to image myocardial inflammation following acute myocardial infarction and takotsubo cardiomyopathy as well as macrophage activity in carotid atherosclerosis and abdominal aortic aneurysms. USPIO-enhanced CMR is emerging as a promising non- 
invasive method of imaging cellular myocardial inflammation providing valuable diagnostic and prognostic information. It could provide valuable information in terms of monitoring disease activity following myocardial infarction or takotsubo cardiomyopathy or in guiding the use and monitoring the effects of novel anti-inflammatory treatments. Utilisation of USPIO's diverse physiological properties in combination with optimisation of CMR sequences will help overcome some of the future challenges, in the hope that translation of USPIO imaging into routine clinical practice might aid patient risk stratification and to targeted individualised patient treatment. 


\section{References}

1. Chovatiya R., Medzhitov R. Stress, inflammation, and defense of homeostasis. Mol Cell 2014;54(2):281-8.

2. Cao DJ. Macrophages in Cardiovascular Homeostasis and Disease. Circulation $2018 ; 138(22): 2452-5$.

3. Bandaru S., Ala C., Salimi R., et al. Targeting Filamin A Reduces Macrophage Activity and Atherosclerosis. Circulation 2019;140(1):67-9.

4. Ma Y., Mouton AJ., Lindsey ML. Cardiac macrophage biology in the steady-state heart, the aging heart, and following myocardial infarction. Transl Res 2018;191:1528.

5. Bjørnerud A., Johansson L. The utility of superparamagnetic contrast agents in MRI: Theoretical consideration and applications in the cardiovascular system. NMR Biomed 2004;17(7):465-77.

6. Dulińska-Litewka J., Łazarczyk A., Hałubiec P., Szafrański O., Karnas K., Karewicz A. Superparamagnetic Iron Oxide Nanoparticles - Current and Prospective Medical Applications. Materials (Basel) 2019;12(4):617.

7. Bietenbeck M., Florian A., Sechtem U., Yilmaz A. The diagnostic value of iron oxide nanoparticles for imaging of myocardial inflammation - Quo vadis? J Cardiovasc Magn Reson 2015;17(1):1-12.

8. Cortajarena AL., Ortega D., Ocampo SM., et al. Engineering Iron Oxide Nanoparticles for Clinical Settings. Nanobiomedicine 2014;1(1):2.

9. Stirrat C., Vesey A., McBride O., et al. Ultra-small superparamagnetic particles of iron oxide in magnetic resonance imaging of cardiovascular disease. J Vasc Diagnostics 2014;2:99-112.

10. Wang YXJ., Hussain SM., Krestin GP. Superparamagnetic iron oxide contrast agents: 
Physicochemical characteristics and applications in MR imaging. Eur Radiol 2001;11(11):2319-31.

11. Bean CP., Livingston JD. Superparamagnetism. J Appl Phys 1959;30(4):S120-9.

12. Weissleder R., Nahrendorf M., Pittet MJ. Imaging macrophages with nanoparticles. Nat Mater 2014;13(2):125-38.

13. Neuwelt EA., Papisov M., Weissleder R., Bogdanov A. Long-circulating iron oxides for MR imaging. Adv Drug Deliv Rev 1995;16(617):321-34.

14. Weissleder R., Elizondo G., Wittenberg J., Rabito C., Bengele H., Josephson L. Ultrasmall Agents for Superparamagnetic of a New Class Iron Oxide : of Contrast Agents for MR Imaging. Radiology 1990;175:489-93.

15. Alam SR., Stirrat C., Spath N., et al. Myocardial inflammation, injury and infarction during on-pump coronary artery bypass graft surgery. J Cardiothorac Surg 2017;12(1):1-10.

16. Stirrat CG., Alam SR., MacGillivray TJ., et al. Ferumoxytol-enhanced magnetic resonance imaging assessing inflammation after myocardial infarction. Heart 2017;103(19):1528-35.

17. Stirrat CG., Alam SR., MacGillivray TJ., et al. Ferumoxytol-enhanced magnetic resonance imaging methodology and normal values at 1.5 and 3T. J Cardiovasc Magn Reson 2016;18(1):1-9.

18. Yilmaz A., Rösch S., Klingel K., et al. Magnetic resonance imaging (MRI) of inflamed myocardium using iron oxide nanoparticles in patients with acute myocardial infarction - Preliminary results. Int J Cardiol 2013;163(2):175-82.

19. Yilmaz A., Dengler MA., Van Der Kuip H., et al. Imaging of myocardial infarction using ultrasmall superparamagnetic iron oxide nanoparticles: A human study using a multi-parametric cardiovascular magnetic resonance imaging approach. Eur Heart J 
$2013 ; 34(6): 462-75$.

20. Sosnovik DE., Nahrendorf M., Deliolanis N., et al. Fluorescence tomography and magnetic resonance imaging of myocardial macrophage infiltration in infarcted myocardium in vivo. Circulation 2007;115(11):1384-91.

21. Montet-Abou K., Daire JL., Hyacinthe JN., et al. In vivo labelling of resting monocytes in the reticuloendothelial system with fluorescent iron oxide nanoparticles prior to injury reveals that they are mobilized to infarcted myocardium. Eur Heart J 2010;31(11):1410-20.

22. Yang Y., Yang Y., Yanasak N., Schumacher A., Hu TCC. Temporal and noninvasive monitoring of inflammatory-cell infiltration to myocardial infarction sites using micrometer-sized iron oxide particles. Magn Reson Med 2010;63(1):33-40.

23. Protti A., Dong X., Andia ME., et al. Assessment of inflammation with a very small iron-oxide particle in a murine model of reperfused myocardial infarction. J Magn Reson Imaging 2014;39(3):598-608.

24. Saleh A., Schroeter M., Jonkmanns C., Hartung HP., Mödder U., Jander S. In vivo MRI of brain inflammation in human ischaemic stroke. Brain 2004;127(7):1670-7.

25. Saleh A., Schroeter M., Ringelstein A., et al. Iron oxide particle-enhanced MRI suggests variability of brain inflammation at early stages after ischemic stroke. Stroke 2007;38(10):2733-7.

26. Wang G., Serkova NJ., Groman E V., Scheinman RI., Simberg D. Feraheme (Ferumoxytol) Is Recognized by Proinflammatory and Anti-inflammatory Macrophages via Scavenger Receptor Type AI/II. Mol Pharm 2019;16(10):4274-81.

27. Messroghli DR., Moon JC., Ferreira VM., et al. Clinical recommendations for cardiovascular magnetic resonance mapping of T1, T2, T2 and extracellular volume: A consensus statement by the Society for Cardiovascular Magnetic Resonance (SCMR) 
endorsed by the European Association for Cardiovascular Imagin. J Cardiovasc Magn Reson 2017;19(1):1-24.

28. Smith GC., Carpenter JP., He T., Alam MH., Firmin DN., Pennell DJ. Value of black blood T2* cardiovascular magnetic resonance. J Cardiovasc Magn Reson 2011;13(1):1-6.

29. Stirrat CG., Alam SR., MacGillivray TJ., et al. Ferumoxytol-enhanced magnetic resonance imaging methodology and normal values at 1.5 and 3T. J Cardiovasc Magn Reson 2016;18(1):1-9.

30. Finn JP., Nguyen KL., Han F., et al. Cardiovascular MRI with ferumoxytol. Clin Radiol 2016;71(8):796-806.

31. Taktak S., Sosnovik D., Cima MJ., Weissleder R., Josephson L. Multiparameter magnetic relaxation switch assays. Anal Chem 2007;79(23):8863-9.

32. Harisinghani M., Dixon T., Saksena M., et al. phy: Imaging Strategies to Optimize the Imag- ing of Lymph Nodes with Ferumoxtran-10 1. Imaging Ther Technol 2004;24(3):867-78.

33. Alam SR., Shah ASV., Richards J., et al. Ultrasmall superparamagnetic particles of iron oxide in patients with acute myocardial infarction early clinical experience. Circ Cardiovasc Imaging 2012;5(5):559-65.

34. Lagan J., Naish J., Clark D., et al. In vivo evidence of chronic myocardial inflammation in ischaemic cardiomyopathy using USPIO enhanced cardiovascular magnetic resonance. Eur Heart J 2017;Abstract 3:693-4.

35. Ferreira VM., Schulz-Menger J., Holmvang G., et al. Cardiovascular Magnetic Resonance in Nonischemic Myocardial Inflammation: Expert Recommendations. J Am Coll Cardiol 2018;72(24):3158-76.

36. Moon H., Park HE., Kang J., et al. Noninvasive assessment of myocardial 
inflammation by cardiovascular magnetic resonance in a rat model of experimental autoimmune myocarditis. Circulation 2012;125(21):2603-12.

37. Stirrat CG., Alam SR., MacGillivray TJ., et al. Ferumoxytol-enhanced magnetic resonance imaging in acute myocarditis. Heart 2018;104(4):300-5.

38. Mahrholdt H., Goedecke C., Wagner A., et al. Cardiovascular Magnetic Resonance Assessment of Human Myocarditis. Circulation 2004;109(10):1250-8.

39. Baughman KL. Diagnosis of myocarditis death of Dallas criteria. Circulation 2006;113(4):593-5.

40. Scally C., Abbas H., Ahearn T., et al. Myocardial and Systemic Inflammation in Acute Stress-Induced (Takotsubo) Cardiomyopathy. Circulation 2019;139(13):1581-92.

41. Abdel-Aty H., Cocker M., Friedrich MG. Myocardial edema is a feature of TakoTsubo cardiomyopathy and is related to the severity of systolic dysfunction: Insights from T2-weighted cardiovascular magnetic resonance. Int J Cardiol 2009;132(2):2913.

42. Eitel I., Lücke C., Grothoff M., et al. Inflammation in takotsubo cardiomyopathy: Insights from cardiovascular magnetic resonance imaging. Eur Radiol 2010;20(2):422-31.

43. Dweck MR., Aikawa E., Newby DE., et al. Noninvasive Molecular Imaging of Disease Activity in Atherosclerosis. Circ Res 2016;119(2):330-40.

44. Corot C., Kolb S., Debatin JF., Vogt P., Ruehm SG. Magnetic Resonance Imaging of Atherosclerotic Plaque With Ultrasmall Superparamagnetic Particles of Iron Oxide in Hyperlipidemic Rabbits. Circulation 2001;103:415-22.

45. Kresse M., Wagner S., Thode K., Dinkelborg L. MR plaque imaging using superparamagnetic iron oxide particles. Proc ISMRM 1998.

46. Tang TY., Howarth SPS., Miller SR., et al. The ATHEROMA (Atorvastatin Therapy: 
Effects on Reduction of Macrophage Activity) Study. J Am Coll Cardiol 2009;53(22):2039-50.

47. Tang T., Howarth SPS., Miller SR., et al. Assessment of inflammatory burden contralateral to the symptomatic carotid stenosis using high-resolution ultrasmall, superparamagnetic iron oxide-enhanced MRI. Stroke 2006;37(9):2266-70.

48. Alam SR., Stirrat C., Richards J., et al. Vascular and plaque imaging with ultrasmall superparamagnetic particles of iron oxide. J Cardiovasc Magn Reson 2015;17(1):83.

49. Tang TY., Howarth SPS., Miller SR., et al. Correlation of carotid atheromatous plaque inflammation using USPIO-enhanced MR imaging with degree of luminal Stenosis. Stroke 2008;39(7):2144-7.

50. Tang TY., Moustafa RR., Howarth SP., et al. Combined PET-FDG and USPIOenhanced MR Imaging in Patients with Symptomatic Moderate Carotid Artery Stenosis. Eur J Vasc Endovasc Surg 2008;36(1):53-5.

51. Smits LP., Tiessens F., He K., et al. Evaluation of ultrasmall superparamagnetic ironoxide ( USPIO ) enhanced MRI with ferumoxytol to quantify arterial wall in fl ammation. Atherosclerosis 2017;263:211-8.

52. Metabolic Imaging in Carotid Atherosclerosis. Available at: https://clinicaltrials.gov/ct2/show/NCT01674257?term=nct01674257\&draw=2\&rank= 1. Accessed November 14, 2019.

53. Howarth SPS., Tang TY., Graves MJ., et al. Non-invasive MR imaging of inflammation in a patient with both asymptomatic carotid atheroma and an abdominal aortic aneurysm: A case report. Ann Surg Innov Res 2007;1(4):1-4.

54. Richards JMJ., Semple SI., MacGillivray TJ., et al. Abdominal aortic aneurysm growth predicted by uptake of ultrasmall superparamagnetic particles of Iron oxide : A pilot study. Circ Cardiovasc Imaging 2011;4(3):274-81. 
55. Newby D., Forsythe R., McBride O., et al. Aortic Wall Inflammation Predicts Abdominal Aortic Aneurysm Expansion, Rupture, and Need for Surgical Repair. Circulation 2017;136(9):787-97.

56. McBride OMB., Joshi N V., Robson JMJ., et al. Positron Emission Tomography and Magnetic Resonance Imaging of Cellular Inflammation in Patients with Abdominal Aortic Aneurysms. Eur J Vasc Endovasc Surg 2016;51(4):518-26.

57. Satomi T., Ogawa M., Mori I., et al. Comparison of contrast agents for atherosclerosis imaging using cultured macrophages: FDG versus ultrasmall superparamagnetic iron oxide. J Nucl Med 2013;54(6):999-1004.

58. Hasan D., Chalouhi N., Jabbour P., et al. Early change in ferumoxytol-enhanced Magnetic Resonance Imaging signal suggests unstable human cerebral aneurysm. A pilot study. Stroke 2013;43(12):3258-65.

59. Hasan DM., Chalouhi N., Jabbour P., et al. Evidence That Acetylsalicylic Acid Attenuates In $\mathrm{fl}$ ammation in the Walls of Human Cerebral Aneurysms : Preliminary Results. J Am Hear Assoc 2013;2:1-6.

60. Shimizu K., Kushamae M., Aoki T. Macrophage imaging of intracranial aneurysms. Neurol Med Chir (Tokyo) 2019;59(7):257-63.

61. Nahrendrof M., Pittet M., Swieski F. Monocytes: protagonists of infarct inflammation and repair. Circulation 2010;121(22):2437-45.

62. Tsujioka H., Imanishi T., Ikejima H., et al. Impact of Heterogeneity of Human Peripheral Blood Monocyte Subsets on Myocardial Salvage in Patients With Primary Acute Myocardial Infarction. J Am Coll Cardiol 2009;54(2):130-8.

63. Schultze JL. Myocardial infarction cell by cell. Nat Immunol 2019;20(1):7-9.

64. Zini C., Venneri MA., Miglietta S., et al. USPIO-labeling in M1 and M2-polarized macrophages: An in vitro study using a clinical magnetic resonance scanner. J Cell 
Physiol 2018;233(8):5823-8.

65. Khaled W., Piraquive J., Leporq B., et al. In vitro distinction between proinflammatory and antiinflammatory macrophages with gadolinium-liposomes and ultrasmall superparamagnetic iron oxide particles at 3.0T. J Magn Reson Imaging 2019;49(4):1166-73.

66. Shekhar A., Heeger P., Reutelingsperger C., et al. Targeted Imaging for Cell Death in Cardiovascular Disorders. JACC Cardiovasc Imaging 2018;11(3):476-93.

67. Bulluck H., Dharmakumar R., Arai AE., Berry C., Hausenloy DJ. Cardiovascular magnetic resonance in acute st-segment-elevation myocardial infarction: Recent advances, controversies, and future directions. Circulation 2018;137(18):1949-64.

68. Stirrat CG., Newby DE., Robson JMJ., Jansen MA. The Use of Superparamagnetic Iron Oxide Nanoparticles to Assess Cardiac Inflammation. Curr Cardiovasc Imaging Rep 2014;7(5):1-8.

69. Schellenberger EA., Sosnovik D., Weissleder R., Josephson L. Magneto/optical annexin V, a multimodal protein. Bioconjug Chem 2004;15(5):1062-7.

70. Sosnovik DE., Garanger E., Aikawa E., et al. Molecular MRI of Cardiomyocyte Apoptosis With Simultaneous Delayed-Enhancement MRI Distinguishes Apoptotic and Necrotic Myocytes In Vivo. Circ Cardiovasc Imaging 2009;2(6):460-7.

71. European Medicines Agency. Rienso: Withdrawal of the application to change the marketing authorisation. 2015. Available at: https://www.ema.europa.eu/en/medicines/human/withdrawn-applications/rienso.

72. European Medicines Agency. Rienso. Withdrawal of marketing authorisation in the European Union. Available at: www.ema.europa.eu/docs/en_GB/document_library/Public_statement/2015/07/WC50 0189474.pdf. 
73. U.S. Food and Drug Administration. FDA Drug Safety Communication: FDA strengthens warnings and changes prescribing instructions to decrease the risk of serious allergic reactions with anemia drug Feraheme (ferumoxytol). Available at: https://www.fda.gov/drugs/drug-safety-and-availability/fda-drug-safetycommunication-fda-strengthens-warnings-and-changes-prescribing-instructionsdecrease. Accessed March 30, 2015.

74. Nguyen K., Yoshida T., Kathuria-prakash N., et al. Multicenter Safety and Practice for Off-Label Diagnostic Use of Ferumoxytol in MRI. Radiology 2019;293(3):554-64.

75. Adkinson NF., Strauss WE., Macdougall IC., et al. Comparative safety of intravenous ferumoxytol versus ferric carboxymaltose in iron deficiency anemia: A randomized trial. Am J Hematol 2018;93(5):683-90.

76. Hope MD., Hope TA., Zhu C., et al. Vascular imaging with ferumoxytol as a contrast agent. Am J Roentgenol 2015;205(3):W366-73.

77. Stoumpos S., Hennessy M., Vesey AT., et al. Ferumoxytol magnetic resonance angiography: a dose-finding study in patients with chronic kidney disease. Eur Radiol 2019;29:3543-52.

78. Nguyen KL., Moriarty JM., Plotnik AN., et al. Ferumoxytol-enhanced MR angiography for vascular access mapping before transcatheter aortic valve replacement in patients with renal impairment: A step toward patient-specific care. Radiology 2018;286(1):326-37.

79. Stoumpos S., Hennessy M., Vesey AT., et al. Ferumoxytol-enhanced magnetic resonance angiography for the assessment of potential kidney transplant recipients. Eur Radiol 2018;28(1):115-23.

80. Toth GB., Varallyay CG., Horvath A., et al. Current and potential imaging applications of ferumoxytol for magnetic resonance imaging. Kidney Int 2017;92(1):47-66. 
81. Knobloch G., Colgan T., Wiens C., et al. Relaxivity of ferumoxytol at 1.5T and 3.0T. Invest Radiol 2018;53(5):257-63.

82. Metz S., Bonaterra G., Rudelius M., Settles M., Rummeny EJ., Daldrup-Link HE. Capacity of human monocytes to phagocytose approved iron oxide MR contrast agents in vitro. Eur Radiol 2004;14(10):1851-8.

83. Neuwelt EA., Hamilton BE., Varallyay CG., et al. Ultrasmall superparamagnetic iron oxides (USPIOs): A future alternative magnetic resonance (MR) contrast agent for patients at risk for nephrogenic systemic fibrosis (NSF)? Kidney Int 2009;75(5):46574.

84. Simon GH., Bauer J., Saborovski O., et al. T1 and T2 relaxivity of intracellular and extracellular USPIO at 1.5T and 3T clinical MR scanning. Eur Radiol 2006;16(3):73845. 


\section{Figure Legends:}

Figure 1: Ferumoxytol-enhanced CMR in patient with myocardial infarction

Panel A: Patient 1 week post left anterior descending artery infarction with extensive, anterior wall, transmural, mid-ventricle LGE on T1-weighted images (left column), homogeneous myocardial T2* values prior to ferumoxytol (middle column), but intense dark ferumoxytol uptake in the region of the infarction, 24 hours post ferumoxytol infusion (right column). Panel B: Same patient with anteroapical, transmural LGE, again homogeneous T2* myocardial values prior to ferumoxytol, but clear ferumoxytol uptake on T2* scanning in the region of the LGE 24 hours after ferumoxytol infusion.

LGE: Late Gadolinium Enhancement

Figure 2: Myocardial edema and cellular inflammation following myocardial infarction Examples of myocardial edema and ferumoxytol enhancement in the infarct area after MI. Three examples of MI (1-anteroseptal, 2-lateral and 3-inferior) demonstrating LGE on T1weighted imaging (A), ferumoxytol enhancement (R2* maps; B, C) and edema (T2 maps; D, E) at early (up to 10 days; B, D) and late (3 months; C, E) post MI. Early inflammation and edema seen on R2* maps (dark region) and T2 maps (light region), respectively, have improved or resolved by 3 months. Reproduced with permission from Stirrat et al. (16) MI: Myocardial Infarction, LGE: Late Gadolinium Enhancement

Figure 3: Ferumoxytol-enhanced CMR in patient with acute myocarditis

Patient with acute myocarditis clearly evident sub-epicardial LGE, inferiorly and inferolaterally on 4-chamber and 3-chamber views (left) but no ferumoxytol uptake (right) 24 hours following ferumoxytol infusion.

LGE: Late Gadolinium Enhancement

Figure 4: Ferumoxytol-enhanced CMR in healthy volunteer

Healthy volunteer with no LGE visible on T1-weighted imaging (left) and homogeneous myocardial signal on T2* (right) 24 hours after ferumoxytol infusion.

\section{Figure 5: Ferumoxytol-enhanced CMR in patient with takotsubo cardiomyopathy}

Mid-cavity short axis views from a patient with acute takotsubo cardiomyopathy demonstrating a $12 \mathrm{~ms}$ change in $\mathrm{T} 2 *$ from pre (left) to post (right) ferumoxytol infusion. In healthy controls the change in $\mathrm{T} 2 *$ is usually $8-9 \mathrm{~ms}$ at $3 \mathrm{~T}$.

\section{Figure 6: Monocyte response in a myocardial infarction mouse model}

An illustration of the biphasic monocyte response after myocardial infarction in a mouse model. The time course of monocyte subset recruitment and their function is depicted in the lower panels

\section{Central Illustration: Imaging cellular inflammation with USPIO-enhanced CMR}

Cardiac magnetic resonance enhanced with ultrasmall superparamagnetic particles of iron oxide allows imaging of myocardial cellular inflammation in myocardial infarction and takotsubo cardiomyopathy but not myocarditis 
Table 1. Summary of characteristics of some ION-based contrast agents commonly used in the studies that we will discuss in this review. Summary of discussed ION-based

contrast agents. Relaxivities are given at $1.5 \mathrm{~T}$ in plasma $(1,2)$ or Ficoll solution $(3)$, at $37{ }^{\circ} \mathrm{C}$.

Table 1: Summary of discussed ION-based contrast agents

\begin{tabular}{|c|c|c|c|c|c|}
\hline Name & Coating & $\begin{array}{l}\text { Size } \\
(\mathrm{nm})\end{array}$ & $\begin{array}{l}\text { Plasma } \\
\text { half-life (h) }\end{array}$ & $\begin{array}{l}\text { r1 relaxivity } \\
\left(\mathrm{mM}^{-1} \mathrm{~s}^{-1}\right)\end{array}$ & $\begin{array}{l}\text { r2 relaxivity } \\
\left(\mathrm{mM}^{-1} \mathrm{~s}^{-1}\right)\end{array}$ \\
\hline Ferumoxytol (Feraheme $\left.{ }^{\mathrm{TM}}\right)$ & $\begin{array}{l}\text { Carboxymethyld } \\
\text { extran }\end{array}$ & $17-31$ & $14-21(80)$ & $19(81)$ & $65(81)$ \\
\hline $\begin{array}{l}\text { Ferucarbotran (SHU 555A, } \\
\text { Resovist) }\end{array}$ & Carboxydextran & $45-60$ & $<1(18)$ & $7(82)$ & $82(82)$ \\
\hline $\begin{array}{l}\text { Ferumoxtran (USPIO, AMI- } \\
\text { 227, NC100150, BMS- } \\
180549)\end{array}$ & Dextran & $17-21$ & $24-36(83)$ & $16(84)$ & $100(84)$ \\
\hline
\end{tabular}

